\title{
Children's emotions and its social meaning
}

\begin{abstract}
The word emotion refers to a broad repertoire of perceptions, expressions of feelings and bodily changes. Emotion is a feeling that is private and subjective, a state of psychological arousal an expression or display of distinctive somatic and autonomic responses and actions commonly "deemed", such as defending or attacking in response to a threat. This paper focuses on selected social stories to be story told to a group of asperger children in Malaysia. Social stories are a tool for teaching social skills to children with autism, asperger syndrome and related disabilities. The selected social stories would be based on Malaysian culture and the children's emotions and conversation were videotaped and analysed its social meaning by using the content analysis approach. There are six basic emotions grouped in three pairs of opposites; joy and sadness, acceptance and disgust and anger and fear. Four asperger syndrome subjects from a school in Malaysia were chosen for the purpose of this study. Three social stories were read to them repeatedly for two hours a day in three weeks. Subsequently, the children were able to use the social stories in their everyday lives.
\end{abstract}

Keyword: Emotion, asperger syndrome, social meaning 